\title{
Repères bibliographiques du dossier « École et religion »
}

\section{Françoise Profit}

\section{OpenEdition}

1 Journals

Édition électronique

URL : http://journals.openedition.org/ries/1539

DOI : 10.4000/ries.1539

ISSN : 2261-4265

Éditeur

Centre international d'études pédagogiques

Édition imprimée

Date de publication : 1 septembre 2004

Pagination : 159-168

ISBN : 978-2-85420-561-8

ISSN : $1254-4590$

\section{Référence électronique}

Françoise Profit, «Repères bibliographiques du dossier « École et religion » », Revue internationale d'éducation de Sèvres [En ligne], 36 | septembre 2004, mis en ligne le 21 novembre 2011, consulté le 03 mai 2019. URL : http://journals.openedition.org/ries/1539; DOI : 10.4000/ries.1539

(C) Tous droits réservés 


\section{Repères \\ bibliographiques}

\section{Françoise Profit}

Cette bibliographie, non exhaustive, reflète le fonds d'ouvrages disponible sur ce sujet au Centre de ressources documentaire du CIEP. Elle présente tout d'abord des études générales portant sur la laïcité et la sociologie des religions, puis un panorama historique des textes législatifs français traitant des relations entre l’Église et l'État. Elle indique ensuite les principaux rapports officiels français sur la question de l'intégration des religions dans la République. Enfin, une dernière partie est consacrée à l'enseignement du fait religieux en France essentiellement, et mentionne également quelques études transnationales.

Bibliographie arrêtée le 10 juin 2004.

\section{Études générales}

\section{Laïcité}

BAUBEROT Jean, Histoire de la lä̈cité en France. PUF (Que sais-je?) n³571, 2003, 128 p.

La France est constitutionnellement une République laïque mais l'histoire de la laïcité est assez peu connue. L'auteur retrace son histoire depuis la Révolution, montre que la laïcité est liée au pacte républicain ; le dernier chapitre est consacré à la crise, de la laïcité depuis les années quatre-vingt et à son renouveau.

\section{BAUMANN Maurice, Le protestantisme et l'école : plaidoyer religieux pour un} nouvel enseignement laïc. Labor et Fides/Genève/Suisse (Protestantismes), 1999, 115 p.

Le protestantisme est à l'origine un des grands promoteurs de l'enseignement laïc ouvert à tous, favorisant l'apprentissage par l'élève de l'autonomie et de la citoyenneté. Comment défendre aujourd'hui cette option? L'auteur donne plusieurs pistes pour élargir les débats sur la transmission des valeurs aux nouvelles générations.

\section{BOUCHET Gérard, Laïcité : textes majeurs pour un débat d'actualité.}

A. Colin/Paris, (Formation des enseignants. Enseigner), 1997, 192 p., index.

La présentation de ces textes sur la laïcité est organisée selon quatre grands axes : les précurseurs (de Socrate à Auguste Comte), les constructeurs (de Edgar Quinet à Alain), les adversaires (de Adolphe Thiers à Jean-Paul II), la continuation du débat (de Gaston Bachelard à Paul Ricoeur).

Cahier la famille et l'école, "Les diversités culturelles : un atout pour la lä̈cité», 2004/05, $n^{\circ} 55,70 p$.

La Fédération des conseils de parents d'élèves (FCPE), organisation laïque, a voulu, lors de son colloque, revenir sur le contexte social et politique du paysage religieux français qui a bien changé depuis la loi de 1905. L'école est le lieu où se sont incarnés les débats. Les intervenants au colloque sont revenus dans un premier temps sur l'histoire de la laïcité et l'élaboration des lois en France. Dans un deuxième temps, l'apport interculturel que transmettent les différentes religions à travers les civilisations et la mixité sociale ont été soulignés. 
COSTA-LASCOUX Jacqueline, Les trois âges de la laïcité. Hachette/Paris, (Questions de politique. Savoirs enjeux débats), 1996, 140 p., bibliogr.

Les trois âges de la laïcité sont la séparation de l'Église et de l'État, la neutralité religieuse, puis la reconnaissance d'une liberté religieuse garantissant le pluralisme. Cet ouvrage analyse aussi le concept de laïcité à la française et propose que cette idée soit celle adoptée pour l'Europe. Il se termine par un débat avec le grand rabbin de France.

\section{GAUCHET Marcel, La religion dans la démocratie. Gallimard/Paris (Folio. Essais), 1998, $n^{\circ} 394,175 p$.}

Pour l'auteur, les relations entre l'État et la religion en France ont changé. Son livre retrace l'histoire de la religion au sein de la République et aborde la sortie de la religion, ce qui ne signifie pas sortie de la croyance religieuse, mais sortie d'un monde où la croyance est structurante et commande la forme politique des sociétés. Il en donne pour preuve l'affaiblissement marqué des Églises établies et des adhésions confessionnelles, tout du moins en Europe.

\section{HAMMEL Jean-Pierre, LADRIERE Muriel, Héritages : la culture occidentale dans ses racines religieuses. Hatier/Paris, 1991, 413 p., ill., index.}

Cet ouvrage retrace l'histoire des religions chrétiennes à travers les âges, puis présente la Bible et les pratiques des religions juive, catholique, protestante et orthodoxe. Les dernières parties sont consacrées aux relations qu'entretiennent les religions avec les arts picturaux et littéraires. Cet ouvrage comporte également des explications du vocabulaire spécifique au religieux.

\section{Les idées en mouvement, "Laïcité : "fait et à faire" ", 1998/04, supplément au} $n^{\circ} 58,80 \mathrm{p}$.

Cet article est entièrement consacré à la laïcité en France et réactive ainsi un débat essentiel à l'idée que l'on se fait de la République. Le débat se situe autour de quatre grands axes : la nouvelle donne religieuse (mutation du catholicisme, émergence de l'islam); la laïcité, une singularité française en Europe; la morale commune dans une société laïque; la nouvelle question scolaire. L'ensemble est complété par une page de bibliographie.

\section{Migrants-Formation, "Religions et intégration", 1990/09, n 82, 184 p.}

Ce numéro, composé de quatre parties, est consacré aux problèmes que soulève le retour du religieux dans la société française. La première partie aborde ce phénomène à travers les pratiques et les croyances des immigrés. Il envisage ensuite l'islam en France et ses différents courants. La troisième partie est consacrée aux autres religions que les immigrés apportent en venant s'installer en France. La dernière partie est consacrée aux problèmes que pose l'introduction, dans un système laïque, de cours d'histoire des religions.

Regards sur l'actualité, "État, lä̈cité, religion : dossier», Documentation française/Paris, 2004, $n^{\circ}$ 298, pp. 5-66.

Ce dossier prend pour point de départ le débat sur l'intégration des populations issues de l'immigration, souvent de culture arabo-musulmane, et revient sur les fondements juridiques de la laïcité en France. La deuxième partie est spécifiquement consacrée au projet de loi relatif à l'application du principe de laïcité dans les établissements scolaires publics français. La troisième partie traite des relations entre l'islam et la République et présente le Conseil français du culte musulman $(\mathrm{CFCM})$ mis en place par les autorités françaises pour aider à régler les problèmes liés à l'exercice du culte musulman. Les deux dernières contributions permettent de faire le point sur la polémique qui oppose partisans et opposants de la révision de la loi de 1905. 
SAMUEL Albert, La lä̈cité : une exigence pour la paix. Chronique socialel Lyon/France (Synthèse. Comprendre la société), 1997, 201 p.

La laïcité n'est ni dépassée ni anachronique. L'actualité mondiale française pose d'une manière aigüe la question des rapports entre l'État et les religions. Le maintien ou l'établissement de nations fondées sur l'égalité des citoyens et non sur la juxtaposition des communautés est en jeu, ainsi que la démocratie et la paix.

\title{
Sociologie des religions : études transnationales
}

\begin{abstract}
Ateliers, "Laïcité et mutations socio-religieuses», Université Charles-de-GaulleLille 3/Lille/France, 1998, $n^{\circ}$ 17, 96 p.

Les mutations sociales et religieuses, le réveil de la querelle scolaire, l'affaire du foulard islamique et l'émergence d'un droit européen sur les religions mettent en question le modèle de la laïcité instauré en France par la loi de 1905. Les articles de ce numéro spécial éclairent cette nouvelle donne en analysant les relations entre l'État et les religions dans d'autres pays européens ou non-européens et conduisent à s'interroger sur la nécessité d'envisager en France un «nouveau pacte laïc».
\end{abstract}

BORNET Philippe, BUBLOZ Yvan, BURGER Maya, et al. L'Europe des religions : éléments d'analyse des champs religieux européens. Peter Lang/Frankfurt am Main/Allemagne, 2004/04, 257 p.

Ouvrage qui regroupe un ensemble de contributions issues de différents pays. Les religions dans l'espace européen sont tout d'abord envisagées sous un angle historique et social : identités musulmanes, laïcisation, panorama des religions. La deuxième partie fait le point sur les recherches en cours. Un chapitre est conscré aux «enseignements orientaux». En conclusion est abordé le problème des religions dans le préambule de la constitution européenne.

Cahiers d'études africaines, "Enseignements », Éditions EHESS, 2003, 456 p.

Ensemble de contributions sur l'enseignement en Afrique, qui abordent en particulier la manière dont s'exprime la spécificité des systèmes éducatifs africains (kenyan, nigérian) face aux politiques de mondialisation et les différents savoirs (écoles coraniques, enseignement islamique, apprentissage) transmis en dehors de l'école en Mauritanie, au Mali, au Maroc, en Afrique orientale. L'ouvrage aborde également la question de l'école lors de guerres civiles avec le cas du Congo et de la Somalie et il présente les politiques éducatives durant la période coloniale en s'appuyant notamment sur l'exemple du Togo; le thème de la scolarisation des filles est ici étudié au travers de l'émergence des premières élites féminines africaines.

CORDELLIER Serge coord. L'islamisme. La Découverte/Paris (Les dossiers de l'état du monde), 1994, 159 p., index, glossaire, bibliogr.

Ce dossier tente d'analyser et de comprendre l'importance prise par les mouvements de l'islam politique dans la dernière décennie en s'appuyant sur l'histoire religieuse, politique et sociale des mondes musulmans.

HUSSER Jean-Marie dir. Religion et modernité. Actes de l'université d'automne de Guebwiller 27-30 octobre 2003, Versailles, Scérén-CRDP de Versailles (Les actes de la DESCO), 2004, 331 p.

Réalisé à la suite de l'université d'automne qui visait à mettre en lumière les différents aspects du rapport des religions à la modernité, l'ouvrage présente en premier lieu un discours critique sur les religions illustré par quatre contributions représentatives des approches philo- 
sophique, historique et sociologique des faits religieux. Les auteurs abordent ensuite la place des religions dans la société sécularisée et s'interrogent sur l'attitude des religions face à la modernité depuis le XIX ${ }^{\mathrm{e}}$ siècle. Différentes contributions envisagent enfin la question du fait religieux dans une perspective pédagogique.

KEPEL Gilles, À l'ouest d'Allah. Seuil/Paris (Points. Essai), 1996, 377 p., index. De l'Amérique à l'Europe, des populations musulmanes se sont installées et on fait souche. Des communautés s'organisent qui mettent en question nos sociétés impuissantes à maitriser les exclusions et leur opposent des croyances et un mode de vie censés fournir une alternative radicale.

KEPEL Gilles, Les banlieues de l'Islam. Seuil/Paris (Points Actuels), 1991, 425 p., index.

Gilles Kepel retrace la progression de l'Islam en France depuis 1926, les querelles qui animent cette communauté plurinationale et les évènements qui la soudent, les problèmes politiques, sociaux, économiques qui l'opposent à la société française ou l'en rapprochent, le regain d'intérêt des jeunes générations pour la religion, le débat entre insertion et assimilation.

LEVEAU Rémy, MOHSEN FINAN Khadija, WIHTOL de WENDEN Catherine, L'Islam en France et en Allemagne : identités et citoyennetés. Documentation française/Paris, Institut français de relations internationales/Paris (Études), 2001, $149 p$.

L'immigration de populations musulmanes en France et en Allemagne provoque des débats politiques au sein des deux pays, notamment en ce qui concerne l'accessibilité à la nationalité du pays. Les traditions des deux pays ont entraîné des mouvements différents d'assimilation : intégration en France, multiculturalisme et communautarisme en Allemagne. Cet ouvrage montre les similitudes et les différences de gestion de l'immigration par les deux pays. De nombreux encadrés ponctuent les articles permettant de faire le point sur des particularités liées à un système ou à un pays.

\section{MENDRAS Henri, L'Europe des Européens : sociologie de l'Europe occidentale.} Gallimard/Paris (Folio. Actuel), 1997, 417 p.

Essai de modélisation de la société européenne qui explique les grandes structures et institutions de l'Europe : religion et système de valeurs, hiérarchies et catégories sociales, famille et parenté, État et capitalisme.

Sociétés contemporaines, "Religions et laïcité en Europe", L'Harmattan/Paris, 2000, $n^{\circ} 37,146$ p., bibliogr.

Les sociologues de la religion observent depuis une trentaine d'années une «dérégulation» de la religion en Europe, qui s'exprime par une perte d'emprise institutionnelle et normative de la religion sur la société, une individualisation des croyances et la fin des identités religieuses héritées. Parallèlement, on assiste à de nouvelles émergences religieuses. Les articles réunis ici traitent de la laïcité et du rôle dévolu à la religion par les Européens. Il est plus spécifiquement question des représentations religieuses dans la Russie soviétique et post-soviétique, de la laïcité récente de l'Espagne et du thème de l'intégration en Grande-Bretagne, aux États-Unis, enfin en France.

WILLAIME Jean-Paul, Europe et religions : les enjeux du XXI siècle. Fayard/ Paris (Les Dieux dans la cité), 2004/04, 377 p., bibliogr.

Partant du constat que l'Europe est construite sur des collectivités politiques mais des rapports singuliers au religieux, l'ouvrage présente la laïcité comme l'un des principes fondamentaux de la construction de l'Europe. Il s'agit d'aborder les relations du politique et du religieux dans 
le cadre d'une réflexion d'ensemble sur la mutation de la modernité, nommée «ultramodernité» et dans laquelle le sacré disparaît, qu'il soit politique, scientifique, religieux ou économique. Une «sécularisation pluraliste» permet à la religion d'avoir un rôle à jouer au même titre que l'autonomie individuelle ou le pluralisme politique. L'auteur aborde la singularité française en présentant les relations entre le religieux et la société. Il envisage le respect de la laïcité comme bien commun européen et la collaboration Églises-État dans l'école comme dans la politique.

\section{France}

\section{Historique des textes législatifs}

Loi relative à l'organisation des cultes du 18 germinal, an X. Site http://www. laicite-laligue.org/La ligue de l'enseignement [en ligne]. [consulté le 17 mai 2004]. www.laicite-laligue.org/laligue/laicite-laligue/pdf/concordat.pdf

Ordonnance du Roi concernant le gouvernement de la Guyane française du 27 août 1828. Site http://www.laicite-laligue.org/La ligue de l'enseignement [en ligne]. [consulté le 01 juin 2004]. www.laicite-laligue.org/laligue/laicite-laligue/pdf/ordo_roi.pdf

Loi du 9 décembre 1905 concernant la séparation des Églises et de l’État. J.O. du 11 décembre 1905, pp. 7205-7209.

Loi du 2 janvier 1907 concernant l'exercice public des cultes. J.O. du 3 janvier 1907, p. 34.

Loi du $1^{\text {er }}$ juin 1924 mettant en vigueur la législation civile française dans les départements du Bas-Rhin, du Haut-Rhin et de la Moselle. J.O. du 3 juin 1924, pp. 5026-5043.

Décret-loi du 16 janvier 1939 concernant l'institution aux colonies de conseils d'administration des missions religieuses. Site http://www.laicite-laligue.org/ La ligue de l'enseignement [en ligne]. [consulté le 17 mai 2004]. Décret-loi dit «décret Mandel ». www.laicite-laligue.org/laligue/laicite-laligue/pdf/decret_loi_16_janv_1939.pdf

Décret-loi du 6 décembre 1939 concernant les conseils d'administration des missions religieuses aux colonies. Site http://www.laicite-laligue.org/La ligue de l'enseignement [en ligne]. [consulté le 17 mai 2004]. Décret-loi dit «décret Mandel». www.laicite-laligue.org/laligue/laicite-laligue/pdf/decret_loi_6_decem_1939.pdf

Circulaire du 12 décembre 1989 du ministre d'État, ministre de l'Éducation nationale, de la jeunesse et des sports concernant la réaffirmation du principe de laïcité et les conditions d'autorisation du port de signes religieux [foulard ou voile islamique et autres signes] par les élèves. J.O. du 15 décembre 1989, pp. 15577-15578. 
Code de l'éducation. Bulletin Officiel du ministère de l'Éducation nationale et du ministère de la Recherche spécial $n^{\circ} 7$ du 13 juillet 2000. Première partie, Livre premier, Titre IV, La laïcité de l'enseignement public, articles L141-1 à L141-6.

Arrêté du 14 janvier 2002 portant création du Comité national de réflexion et de propositions sur la laïcité à l'école. J.O. nº 20 du 24 janvier 2002, p. 1612.

LOI n ${ }^{\circ}$ 2004-228 du 15 mars 2004 encadrant, en application du principe de laïcité, le port de signes ou de tenues manifestant une appartenance religieuse dans les écoles, collèges et lycées publics. J.O. nº6 du 17 mars 2004, p. 5190.

\section{Textes officiels, rapports}

Commission nationale consultative des droits de l'homme, La laïcité aujourd'hui : rapport d'étape, 2003.

Site de la Documentation française [en ligne]. Paris : Commission nationale consultative des droits de l'homme, 2003 [consulté le 17 mai 2004]. 21 p. www.ladocumentationfrancaise. fr/brp/notices/034000728.shtml

Conseil d'État, Rapport public 2004. Jurisprudences et avis de 2003; un siècle de laïcité.

«L'objectif était de faire le bilan de cent ans d'application de la loi de 1905 et plus largement du principe de laïcité. En se gardant bien de chercher à établir une définition précise du concept de laïcité, le rapport s'efforce d'appréhender son contenu, sous trois aspects : laïcité et neutralité, laïcité et liberté religieuse et laïcité et pluralisme. L'école est un lieu révélateur des difficultés que peut poser la cohabitation entre croyances. La laïcité de l'enseignement public impose la neutralité des programmes, comme celle des enseignants, corollaire logique de la neutralité du service public. Mais l'État ne peut ignorer le droit à l'instruction religieuse des enfants et la question du "jour de catéchisme" est sensible. La délicate question du port de signes d'appartenance religieuse à l'école est également évoquée.» [Résumé d'après la présentation du rapport en ligne] : http://www.conseil-etat.fr/ce/rappor/index_ra_li0402.shtml

\section{DEBRAY Régis, L'enseignement du fait religieux dans l'école lä̈que. Rapport} au ministre de l'Éducation nationale. Odile Jacob/Paris, SCEREN: Services Culture Éditions Ressources pour l'Éducation Nationale/Paris, 2002, 59 p.

Sans remettre en cause l'esprit de laïcité, et en écartant tout enseignement religieux, l'auteur, dans ce court ouvrage, vient soumettre au ministre un certain nombre de réflexions, propositions et recommandations pour intégrer dans le cadre des disciplines existantes l'enseignement du fait religieux. www.ladocumentationfrancaise.fr/brp/notices/024000544.shtml

DEBRÉ Jean-Louis, Assemblée nationale, La laïcité à l'école : un principe républicain à réaffirmer. Rapport fait au nom de la mission d'information sur la question du port des signes religieux à l'école. Assemblée nationale/Paris, 2003, $n^{\circ} 1275$, tome 2, $658 p$.

Le second tome du rapport sur la laïcité à l'école se fait l'écho des nombreuses auditions qui ont permis un examen progressif et approfondi de tous les aspects du problème du port des signes religieux à l'école. Les auditions sont présentées dans l'ordre chronologique des séances tenues par la mission. 
Haut Conseil à l'intégration, L'Islam dans la République : rapport au Premier ministre. Documentation française/Paris, 2000/11, pp. 1-8, 48-55, 72-86 téléchargées.

Ce rapport porte sur l'islam et ses manifestations en France. Après un premier chapitre consacré à la loi de 1905, établissant le principe de séparation des Églises et de l'État, le rapport comporte quelques pages aussi sur la vie à l'école (cantine, foulard, rythmes scolaires, comportement...) et sur les relations entre l'institution scolaire et les élèves se réclamant de l'islam. L'intégralité de ce rapport peut être consulté sur le site de la Documentation française : http://www.ladocfrancaise.gouv.fr/BRP/

Sénat. Législation comparée/Paris, Le port du foulard islamique à l'école. Sénat/Paris (Législation comparée), 2003/11, $n^{\circ}$ LC 128, 22 p.

Cette étude comparative examine la manière dont six pays européens autorisent les élèves de confession musulmane à porter le foulard dans l'enceinte d'un établissement scolaire. À la suite d'une note de synthèse qui met en exergue les points saillants du débat dans les pays concernés, l'étude offre une fiche par pays qui comprend des informations législatives, les points de conflit éventuels ainsi que le débat sociétal que soulève cette question. http://www.senat. fr/lc/lc128/lc128.pdf

STASI Bernard, Rapport au Président de la République remis le 11 décembre 2003, Commission de réflexion sur l'application du principe de laïcité dans la République.

Site de la Documentation française [en ligne]. Paris : La Documentation française, 2004 [consulté le 17 mai 2004]. 164 p.

La première partie du rapport expose les réformes proposées par le médiateur de la République d'un point de vue législatif. Le rapport revient également sur le rôle du médiateur lorsqu'il est sollicité par ses homologues étrangers et par différents organismes nationaux et internationaux chargés de la promotion et de la protection des droits de l'homme et du citoyen. Le rapport fait ensuite le point sur les délégués du médiateur à l'échelon local, l'évolution de leurs missions ainsi que les perspectives que le médiateur de la République propose de donner à leur présence auprès du public et des administrations locales. [Résumé d'après la présentation du rapport en ligne]. www.ladocumentationfrancaise.fr/brp/notices/044000099.shtml

\section{L'enseignement du fait religieux}

\section{L'enseignement du fait religieux en France}

Administration et éducation, "De l'école et des valeurs» : dossier, 2003, $n^{\circ} 100$, p. 63-131.

Ce numéro consacré à l'école et ses valeurs rassemble plusieurs contributions qui éclairent les multiples aspects de cette question. Le premier article expose dans une perspective historique les valeurs morales qui sous-tendent l'institution scolaire et leur nécessaire évolution. Le second article aborde d'un point de vue philosophique les questions d'égalité, d'autorité et de liberté de l'individu à l'école et montre l'intérêt de ces questions au sein de l'institution scolaire. Les trois autres contributions évoquent les valeurs transmissibles et les modalités de transmission de ces valeurs. 
ALLIEU Nicole, Laïcité et culture religieuse à l'école. ESF/Paris (Pratiques et enjeux pédagogiques), 1996, 124 p., bibliogr.

Faut-il réintroduire l'instruction morale à l'école? La culture religieuse dans le cadre d'une école laïque peut-elle permettre cette réintroduction? Qui doit alors en être responsable et sous l'autorité de qui ? Ces questions conduisent l'auteur à s'interroger sur le modèle de laïcité sur lequel fonctionne l'école aujourd'hui afin de voir la place que pourrait avoir la culture religieuse dans la formation des jeunes. Elle propose également des pistes d'activités éducatives possibles.

AZRIA Régine, Le fait religieux en France. Documentation photographique (Le dossier), 2003, 54 p.

Dans ce dossier, Régine Azria étudie la place du fait religieux dans la société française contemporaine. Se limitant aux trois monothéisme (judaïsme, christianisme, islam), elle dégage les évolutions, pointe les éléments marquants au sein de chaque famille confessionnelle à travers des textes et des documents photographiques. [résumé d'après le site].

\section{BOESPFLUG François dir., MARTINI Evelyne dir., S'initier aux religions : une expérience de formation continue dans l'enseignement public (1995-1999). Cerf/Paris, 1999, 242 p.}

Bilan du stage de formation continue mené de 1995 à 1999 auprès de plus de quatre cents participants appartenant au personnel de l'Éducation nationale. Ce stage avait pour objectif de transmettre une information sur les cinq grandes religions du monde contemporain. L'originalité de cette expérience tient à sa durée, à la demande qu'elle a suscitée et aux pistes ouvertes pour combattre la perte des clés de la culture religieuse. Elle contribue ainsi au débat sur l'enseignement des religions instauré en France depuis une dizaine d'années.

CRDP/Caen/France, CRDP/Besançon/France, Pour enseigner les origines chrétiennes (Histoire des religions), 1996, 205 p., ill. d'après une Université académique organisée les 23-25 octobre 1995 par la MAFPEN de Caen.

Cinq spécialistes apportent leurs réponses à des questions essentielles sur les origines chrétiennes. L'ouvrage propose également une méthodologie d'enseignement des religions adaptée au niveau secondaire, des exemples de travaux pratiques et des séquences pédagogiques.

CRDP : Centre régional de documentation pédagogique/Besançon/France, Enseigner l'histoire des religions dans une démarche laïque : représentations, perspectives, organisation des apprentissages. CRDP/Besançon/France (Documents actes et rapports pour l'éducation) 1992, 347 p.

Actes du colloque international de Besançon, 20-21 novembre 1991. Les contributions au colloque retranscrites dans cet ouvrage tentent de répondre aux problèmes que soulève l'enseignement des religions en classe dans le respect de la laïcité. Le premier atelier aborde les liens entre l'histoire des religions, la culture religieuse et l'enseignement des religions. Le second pose la question de l'approche disciplinaire, pluridisciplinaire ou transdisciplinaire. Le troisième atelier examine la formation des enseignants tandis que le quatrième atelier offre quelques propositions pour renouveler l'enseignement des religions.

\section{DAHERON Blandine, Les religions au collège et au lycée : qu'apprennent nos enfants? Bayard/Paris, 2004, 208 p., bibliogr.}

L'auteur décrit la mise en oeuvre des programmes dans les manuels scolaires pour chacune des disciplines histoire, français, langues, arts plastiques et philosophie et tente de repérer l'angle par lequel est abordé le fait religieux. Grâce à une enquête sur le terrain, l'auteur met 
en lumière la manière dont cet enseignement est vécu et cerne les difficultés et les risques ainsi que les enjeux majeurs d'un tel enseignement.

\section{DEBRAY Régis, DELUMEAU Jean, LANGLOIS Claude, et al., L'enseignement du fait religieux. CRDP/Versailles/France (Les actes de la Desco), 2003, 375 p.}

A la suite d'une des recommandations du rapport de Régis Debray, un séminaire national interdisciplinaire sur l'enseignement du fait religieux s'est tenu à Paris en novembre 2002. Chercheurs, spécialistes d'histoire des religions, de sociologie, de sciences politiques et cadres de l'Éducation nationale se sont réunis pour débattre de l'enseignement du fait religieux dans une perspective pluridisciplinaire afin de permettre à tous les acteurs concernés d'enrichir leur réflexion sur cette question et les aider à construire une pédagogie. Les trois premières parties définissent et analysent le fait religieux dans une approche historique, sociologique et politique. Dans la suite de l'ouvrage, les différentes contributions étudient l'enseignement du fait religieux à partir de textes et d'œuvres artistiques.

\section{FERJANI Mohamed-Chérif, CUSENIER Dominique, Les voies de l'Islam : approche lä̈que des faits islamiques. CRDP/Besançon/France, Cerf/Paris (Histoire des religions), 1996, 267 p., ill. \\ L'auteur définit les conceptions et les pratiques des différentes composantes de l'islam. Une seconde partie propose des outils et des séquences pédagogiques correspondant aux programmes d'enseignement de l'histoire et tenant compte des représentations des élèves.}

\section{FOEHRLE Roger, L'islam pour les profs : recherches pédagogiques. Karthala/ Paris, 1992, 242 p., bibliogr.}

Chaque concept fondamental de l'islam est ici analysé, de façon à créer pour l'enseignant, le formateur ou le lecteur un véritable outil de travail. Pour chacun d'entre eux, un relevé des termes traitant de l'islam dans les ouvrages scolaires a été effectué. Le deuxième volet expose la compréhension musulmane des concepts étudiés.

\section{MESSNER Francis, WOEHRLING Jean-Marie, Les statuts de l'enseignement religieux. Le Cerf/Paris (Droit des religions), 1996, 202 p.}

Cet ouvrage regroupe un ensemble de contributions qui traitent de l'enseignement religieux en France d'un point de vue législatif et historique. Il aborde également les relations entre l'enseignement religieux confessionnel et les écoles publiques. L'exception du droit local d'Alsace-Moselle est souligné. Un volet comparatif présente les statuts de l'enseignement religieux au Luxembourg et en Belgique.

Regards sur l'actualité, "Laïcité religions sectes spiritualités», 1999/03, n²49, pp. 39-51.

Entretien avec Jean-Michel Belorgey sur trois débats : celui sur la laïcité et ses implications, celui sur la place de l'islam dans la société française d'aujourd'hui et celui sur les sectes.

La Revue de l'Inspection générale, "École et République : dossier », 2004/02, n 1, pp. 4-99.

Après avoir replacé dans un contexte historique l'École dans la République, le dossier envisage les relations entre professeurs et République, ce qu'on entend par savoir républicain, les relations entre l'école et l'islam et les liens entre école républicaine et école démocratique. La deuxième partie du dossier offre des regards sur les relations qu'entretiennent les religions et l'école en Allemagne et en Angleterre. Un entretien avec Marcel Gauchet apporte un éclairage philosophique. 
Revue française de pédagogie, "École et culture religieuse», 1998/12, n²125, pp. 5-41, tabl., graph., bibliogr.

Trois contributions étudient les relations entre culture religieuse et éducation. La première la considère d'un point de vue socio-historique, la seconde s'intéresse aux manuels scolaires et à la façon dont ils traitent les faits religieux. Le troisième article présente dans le contexte suisse, les perceptions et les attentes des acteurs du monde éducatif.

\section{Enseignement du fait religieux : études transnationales}

BRODEUR Raymond dir., ROUTHIER Gilles dir., CAULIER Brigitte, L'enseignement religieux : questions actuelles. Le Cerf/Paris, 1996, 162 p.

Journées d'études du groupe de recherche en histoire de l'enseignement religieux de l'Université de Laval. Chaque intervenant rend compte, dans un premier temps, de l'organisation institutionnelle de l'enseignement religieux. Puis chacun fait part des problèmes d'actualité rencontrés: programmes d'enseignement, lieux d'enseignement, manuels et formation des enseignants. Une typologie est dressée afin de permettre à chacun de se situer dans les différentes situations. La seconde partie de l'ouvrage envisage l'avenir de l'enseignement religieux à travers quatre points de vue : mise à distance du modèle du catéchisme, crédibilité d'une parole croyante de nos jours, pédagogie de la foi, enseignements de Vatican II.

Bruxelles/Parlement européen, BOURDARA Kelly, CUATRECASAS Llibert, MONTFORT Elisabeth dir., et al., Dieu a-t-il sa place en Europe? Liberté politique et liberté religieuse dans le traité fondateur de l'Europe réunifiée. François-Xavier De Guibert (Liberté politique), 2003, 290 p.

Actes du colloque de Bruxelles - Parlement européen - 3 avril 2003. Le texte déposé par la Convention pour l'avenir de l'Europe doit être adopté par la Conférence intergouvernementale puis par chacun des États membres avant son application. Dans cette proposition de texte, il n'est pas fait mention de Dieu et une seule référence est faite à l'héritage chrétien. La réflexion engagée au cours de ce colloque pose le problème de la relation entre le politique et le religieux. Il examine la construction d'une Europe de demain qui, dans la Constitution, ne ferait pas référence de manière explicite aux origines et au patrimoine chrétien dont l'entité géopolitique en construction est issue.

WATSON Brenda, The effective teaching of religious education. Longman/ Londres/Royaume-Uni (The effective teacher series), 1993, 222 p., bibliographie. L'enseignement du religieux est complexe et controversé. Il soulève de nombreuses questions auquel l'ouvrage tente de répondre: pourquoi enseigner les religions dans des sociétés sécularisées? Qui peut les enseigner et comment? 